\title{
Accuracy of Sentinel Lymph Node Biopsy in Breast Cancer Using Methylene Blue Dye Technique in a Developing Country
}

\author{
Navin Sookar ${ }^{1 *}$, Neesha Sookar ${ }^{2}$ and Jameel Ali ${ }^{1}$ \\ ${ }^{1}$ Department of Women's Health, St. James Medical Complex, Trinidad and Tobago \\ ${ }^{2}$ Department of Emergency Medicine, Sangre Grande General Hospital, Trinidad and Tobago
}

\begin{abstract}
Introduction: Accurate assessment of the status of the axillary lymph nodes is crucial in staging, planning treatment and determining prognosis in breast cancer. Traditionally this involved total axillary dissection. Recently, in order to decrease morbidity, the technique of Sentinel Lymph Node Biopsy (SLNB) has virtually replaced standard Axillary Lymph Node Dissection (ALND) in T1 and T2 disease with non-palpable axillary lymph nodes. The two commonly applied techniques for SLNB are radio-colloid and dye injection. In our low resource environment, we planned to use the methylene blue dye injection technique to determine the percentages of false negative as well as true positive and true negatives in order to judge the accuracy and guide applicability of this technique in our setting.
\end{abstract}

Methods: We prospectively analysed data from consecutive proven T1 and T2 female breast cancer patients with non-palpable axillary lymph nodes between January and December 2018 at our institution. Exclusion criteria were patients receiving neo-adjuvant treatment, previous breast or axillary radiation, previous axillary surgery and multi-focality. All patients meeting inclusion criteria were assessed by determining the percentage of true positive and negative as well as false negative (by comparing histology of the excised sentinel lymph node with the histology of at least 10 non-sentinel nodes on permanent sections). Demographics such as age of patient, grade of carcinoma, histological subtype and size of tumours were also analysed.

Results: 47 patients were studied. T1 stage accounted for $24 \%$ of the patients and T2 for $76 \%$. The incidence of axillary metastases (both sentinel and axillary nodes) was found to be $57 \%$ (27 of 47). All patients with positive sentinel nodes had at least 2 positive non-sentinel axillary nodes. True negative was $100 \%$ ( 20 of 20 ) and true positive was also $100 \%$ ( 27 of 27 ). There were no false negatives.

Conclusion: In comparison with other SLNB validation studies, we report a very accurate technique of SLNB in our setting. Our data suggest SLNB using methylene blue dye can be used as a reliable alternative to the costly technique of radioactive colloid.

\section{Introduction}

Worldwide, breast cancer is the most frequently diagnosed malignancy and the leading cause of cancer mortality in females [1]. Additionally, it is the leading cause of death in women ages 40 to 49 years. Invasive breast cancer is treated with a multifaceted approach involving primary surgical intervention (wide local excision or mastectomy followed by surgical assessment of the axillary lymph nodes). Adjuvant treatment with radiotherapy and chemotherapy are administered according to standard criteria.

The status of the axillary lymph nodes is one of the most important prognostic factors in guiding staging and treatment of breast cancer. Various non-surgical methods such as ultrasonography, CT Scan, PET Scan and Fine Needle Aspiration Cytology (FNAC) of axillary lymph nodes have all been shown to have poor predictive value in the evaluation of axillary nodal disease [2]. Essentially, axillary nodal staging is best accomplished surgically either by complete Axillary Lymph Node Dissection (ALND) or by Sentinel Lymph Node Biopsy (SLNB). SLNB has been accepted as the standard of care for staging in clinically node-negative (cN0) breast cancer. The morbidity of SLNB is minimal compared to the $15 \%-20 \%$ complication rate which is associated with ALND [3]. The benefits of SLNB include a decreased incidence of postoperative complications specifically lymphedema, paraesthesia and nerve damage compared to formal ALND.
Adequate evaluation of the axilla prior to surgery using clinical examination as well as imaging modalities is very important. Patients with established contraindications (especially clinical node positive disease - cN1-3) for SLNB should undergo ALND. The role and use of SLNB in patients undergoing neoadjuvant chemotherapy (NACT) is currently debated and needs to be further evaluated in clinical trials.

SLNB can be performed with either blue dye and/or radioactive colloid. Multiple fine-cut sections of the sentinel node at $2 \mathrm{~mm}$ slices are prepared for microscopic analysis. This is the widely accepted method for histological analysis of SLN [4].

A review of the literature shows that sentinel lymph nodes are identified in roughly $92 \%$ of cases using the blue dye and up to $98 \%$ when using a combination of blue dye and radioactive colloid. It carries

${ }^{\star}$ Correspondence to: Navin Sookar, Department of Women's Health, St. James Medical Complex, 112 Western Main Road, St. James, Trinidad and Tobago, Tel: (868)-688-6941; E-mail: navin_shawn@hotmail.com

Key words: Sentinel Lymph Node Biopsy (SLNB), Axillary Lymph Node Dissection (ALND), methylene blue dye

Received: December 07, 2018; Accepted: December 18, 2018; Published: December 20, 2018 
a false negative rate of between 5 to $10 \%$ [5]. A false negative sentinel node is defined as a sentinel node which is negative for malignancy, but the rest of axillary lymph nodes demonstrates metastatic cancer. It has been postulated that the cause for false negative sentinel lymph nodes may be lymphatics that have become occluded with malignant tissue or from fibrosis due to prior surgery and/or radiation to the breast.

As reported in the literature, the use of radioactive colloids is an alternative to blue dye. Access to radioactive tracers, cost of nuclear medical services and the availability of gamma probes are the major hurdles in the application of this technique in our resource-depleted setting. The aim of our study was to determine the accuracy of sentinel node biopsy with the use of methylene blue dye using established formal histological analysis of the lymph nodes.

\section{Methods}

Forty-seven consecutive patients with invasive carcinoma of the breast between January to December 2018 were included in the final analysis.

All candidates met the following inclusion criteria: Clinically Node Negative Disease, T1 and T2 breast tumour size, no prior history of breast or axillary surgery, no prior radiotherapy, no NACT and no evidence of metastatic disease on presentation.

We injected $5 \mathrm{~mL}$ of $1 \%$ methylene blue dye of at the tumour periphery, at the palpable edge of the tumour or biopsy cavity.
Following injection, the breast was gently massaged for five minutes to facilitate lymphatic drainage into the lymph node basin. An incision was made extending medial to the border of the pectoralis major muscle. Dissection was used to identify the dye-filled lymphatic channel which was then followed distally until a blue-stained node is seen. The sentinel node is defined as the bluest staining node as evident on gross visual inspection. Dissection up to level 2 axillary lymph nodes was then undertaken.

\section{Results}

For the purpose of our study, we used the American Joint Committee on Carcinoma (AJCC) classification of nodal metastases which is based on size. Macro-metastases were defined as tumour deposits $>2 \mathrm{~mm}$ and micro-metastases as size between $0.2 \mathrm{~mm}$ and $2 \mathrm{~mm}$.

- True positive is defined as a sentinel node which was positive for metastasis and the subsequent axillary nodes were either positive or negative. In the latter, the sentinel node is the only positive node identified. However, in our study, all positive sentinel nodes were associated with other positive axillary nodes.

- True negative is defined as a sentinel node which was negative for metastasis and the subsequent axillary nodes were also negative.

- False negative is defined as a sentinel node which was negative for metastasis and at least one subsequent axillary node was positive (Table 1).

Table 1. Patients and their demographics along with various histopathological criteria

\begin{tabular}{|c|c|c|c|c|c|c|c|c|}
\hline Patient Initials & Age & Tumour Grade & Histology & Tumour Size (T) & Type of Surgery & $\begin{array}{l}\text { Sentinel Lymph } \\
\text { Nodes }\end{array}$ & $\begin{array}{l}\text { Axillary Lymph } \\
\text { Nodes }\end{array}$ & $\begin{array}{c}\text { Final TNM } \\
\text { Staging }\end{array}$ \\
\hline RT & 45 & 2 & DUCTAL & $4.7 \mathrm{~cm}$ & MXT & Positive & Positive & T2N2M0 \\
\hline $\mathrm{T} 2$ & 73 & 2 & DUCTAL & $4.0 \mathrm{~cm}$ & WLE & Positive & Positive & T2N2M0 \\
\hline OG & 35 & 2 & DUCTAL & $3.5 \mathrm{~cm}$ & WLE & Positive & Positive & $\mathrm{T} 2 \mathrm{~N} 2 \mathrm{M} 0$ \\
\hline $\mathrm{CM}$ & 44 & 2 & DUCTAL & $1.3 \mathrm{~cm}$ & WLE & Positive & Positive & T1N2M0 \\
\hline $\mathrm{PC}$ & 79 & 2 & DUCTAL & $2.8 \mathrm{~cm}$ & MXT & Positive & Positive & T2N1M0 \\
\hline SS & 37 & 2 & DUCTAL & $4.7 \mathrm{~cm}$ & MXT & Positive & Positive & T2N2M0 \\
\hline DL & 62 & 2 & DUCTAL & $4.6 \mathrm{~cm}$ & MXT & Positive & Positive & T2N2M1 \\
\hline $\mathrm{RF}$ & 56 & 2 & DUCTAL & $2.3 \mathrm{~cm}$ & WLE & Positive & Positive & T2N1M0 \\
\hline LP & 43 & 2 & LOBULAR & $3.4 \mathrm{~cm}$ & WLE & Positive & Positive & T2N2M0 \\
\hline DA & 65 & 1 & DUCTAL & $4.8 \mathrm{~cm}$ & MXT & Positive & Positive & T2N1M0 \\
\hline FQ & 70 & 2 & DUCTAL & $4.3 \mathrm{~cm}$ & MXT & Positive & Positive & T2N1M0 \\
\hline FJ & 62 & 1 & DUCTAL & $4.6 \mathrm{~cm}$ & MXT & Positive & Positive & T2N1M0 \\
\hline PO & 63 & 1 & DUCTAL & $2.8 \mathrm{~cm}$ & MXT & Positive & Positive & T2N2M0 \\
\hline DR & 45 & 2 & DUCTAL & $1.6 \mathrm{~cm}$ & WLE & Positive & Positive & T1N1M0 \\
\hline TO & 56 & 2 & DUCTAL & $3.1 \mathrm{~cm}$ & WLE & Positive & Positive & T2N2M0 \\
\hline VR & 65 & 1 & DUCTAL & $4.8 \mathrm{~cm}$ & WLE & Positive & Positive & T2N1M0 \\
\hline TG & 58 & 3 & DUCTAL & $4.6 \mathrm{~cm}$ & MXT & Positive & Positive & T2N1M0 \\
\hline DP & 57 & 1 & DUCTAL & $3.1 \mathrm{~cm}$ & WLE & Positive & Positive & T2N1M0 \\
\hline LA & 79 & 2 & LOBULAR & $4.8 \mathrm{~cm}$ & MXT & Positive & Positive & T2N3M1 \\
\hline EB & 75 & 3 & DUCTAL & $3.0 \mathrm{~cm}$ & WLE & Positive & Positive & T2N1M0 \\
\hline NO & 50 & 1 & DUCTAL & $4.6 \mathrm{~cm}$ & MXT & Positive & Positive & T2N2M0 \\
\hline YP & 56 & 3 & DUCTAL & $3.1 \mathrm{~cm}$ & MXT & Positive & Positive & T2N3M0 \\
\hline $\mathrm{AZ}$ & 60 & 1 & DUCTAL & $4.1 \mathrm{~cm}$ & MXT & Positive & Positive & T2N2M0 \\
\hline $\mathrm{HL}$ & 64 & 2 & DUCTAL & $4.5 \mathrm{~cm}$ & MXT & Positive & Positive & T2N3M0 \\
\hline $\mathrm{AM}$ & 70 & 3 & DUCTAL & $1.9 \mathrm{~cm}$ & WLE & Positive & Positive & T1N1M0 \\
\hline $\mathrm{AR}$ & 41 & 2 & DUCTAL & $4.3 \mathrm{~cm}$ & MXT & Positive & Positive & T2N3M0 \\
\hline JA & 40 & 2 & DUCTAL & $4.7 \mathrm{~cm}$ & MXT & Positive & Positive & T2N3M0 \\
\hline LW & 53 & 1 & DUCTAL & $4.4 \mathrm{~cm}$ & MXT & Negative & Negative & T2N0M0 \\
\hline MR & 47 & 2 & DUCTAL & $2.6 \mathrm{~cm}$ & MXT & Negative & Negative & T2N0M0 \\
\hline MF & 63 & 3 & DUCTAL & $3.3 \mathrm{~cm}$ & MXT & Negative & Negative & T2N0M0 \\
\hline $\mathrm{KM}$ & 67 & 2 & DUCTAL & $1.4 \mathrm{~cm}$ & WLE & Negative & Negative & T1N0M0 \\
\hline AP & 56 & 3 & DUCTAL & $3.4 \mathrm{~cm}$ & WLE & Negative & Negative & T2N0M0 \\
\hline
\end{tabular}




\begin{tabular}{|c|c|c|c|c|c|c|c|c|}
\hline DR & 41 & 1 & DUCTAL & $4.6 \mathrm{~cm}$ & MXT & Negative & Negative & T2N0M0 \\
\hline JK & 36 & 1 & DUCTAL & $4.5 \mathrm{~cm}$ & MXT & Negative & Negative & T2N0M0 \\
\hline $\mathrm{HH}$ & 53 & 2 & DUCTAL & $4.6 \mathrm{~cm}$ & MXT & Negative & Negative & T2N0M0 \\
\hline FB & 55 & 3 & DUCTAL & $3.9 \mathrm{~cm}$ & MXT & Negative & Negative & T2N0M0 \\
\hline GR & 47 & 2 & DUCTAL & $2.9 \mathrm{~cm}$ & WLE & Negative & Negative & T2N0M0 \\
\hline ID & 55 & 2 & DUCTAL & $3.4 \mathrm{~cm}$ & MXT & Negative & Negative & T2N0M0 \\
\hline MD & 78 & 2 & DUCTAL & $3.6 \mathrm{~cm}$ & WLE & Negative & Negative & T2N0M0 \\
\hline PH & 47 & 2 & DUCTAL & $3.3 \mathrm{~cm}$ & WLE & Negative & Negative & T2N0M0 \\
\hline MA & 76 & 2 & DUCTAL & $1.8 \mathrm{~cm}$ & WLE & Negative & Negative & T1N0M0 \\
\hline VH & 78 & 2 & DUCTAL & $1.7 \mathrm{~cm}$ & MXT & Negative & Negative & T1N0M0 \\
\hline SR & 61 & 1 & DUCTAL & $1.8 \mathrm{~cm}$ & MXT & Negative & Negative & T1N0M0 \\
\hline EP & 48 & 2 & DUCTAL & $1.6 \mathrm{~cm}$ & WLE & Negative & Negative & T1N0M0 \\
\hline AP & 37 & 3 & DUCTAL & $1.3 \mathrm{~cm}$ & WLE & Negative & Negative & T1N0M0 \\
\hline MB & 78 & 2 & DUCTAL & $1.3 \mathrm{~cm}$ & WLE & Negative & Negative & T1N0M0 \\
\hline $\mathrm{TP}$ & 65 & 1 & DUCTAL & $1.2 \mathrm{~cm}$ & WLE & Negative & Negative & T1N0M0 \\
\hline
\end{tabular}

Legend:

» The median patient age was 57 years (35-79).

"The median pathological tumour size (T) was $3.4 \mathrm{~cm}(1.3 \mathrm{~cm}-4.8 \mathrm{~cm})$.

» Identification rate of SLN was $100 \%$.

» The median number of the identified SNs was 2 (1-6).

" The incidence of axillary metastases (sentinel and non-sentinel nodes) was found to be $57 \%$ (27 of 47 ) of which $79 \%$ of them were macro-metastases.

» True negative rate was $100 \%$ (20 of 20$)$. False negative rate was $0 \%$.

» True positive rate was $100 \%$ (27 of 27). All patients with positive sentinel nodes had at least one additional positive axillary node.

$»$ SLNB accuracy was $100 \%$ (47 of 47 ).

"T1 stage accounted for $24 \%$ (11 of 47$)$ of the patients and $\mathrm{T} 2$ for $76 \%$ (36 of 47$)$

》55\% (26 pf 47) underwent Mastectomy (MXT) while 45\% (21 of 47) had wide local excision (WLE).

There were no anaphylactic reactions, and no cases of skin necrosis during our study.

\section{Discussion}

Axillary lymph node status is one of the strongest prognostic indicators in women with early breast cancer and Sentinel Lymph Node Biopsy (SLNB) has been established as the gold standard in the assessment of metastatic spread to the axillary lymph nodes.

The sentinel node technique was first reported by Morton et al. [6]. The sentinel lymph node is defined as the first node(s) that drains the lymphatics from a particular site before draining into subsequent lymph nodes (non-sentinel nodes). This technique has since been expanded and has been validated for use in breast cancer. A landmark study in 1994 by Giuliano et al. indicated that SLN in breast cancer can accurately identify the status of the entire axillary lymph nodes in terms of detecting nodal metastasis [7]. Its use is currently being investigated in the field of colon, renal cell, penile and prostate cancer [8].

Formal ALND is associated with significant morbidity with lymphedema rate reported as high as $20 \%-30 \%$. Sentinel lymph node biopsy (SLNB) is a proven and more therapeutically beneficial alternative to complete Axillary Lymph Node Dissection (ALND) in clinically node-negative patients. Multiple research papers have recommended its use in the initial evaluation of the axilla because of its accuracy and limited morbidity. As in our present report, many authors have also suggested the use of methylene blue alone for sentinel node biopsy without the need for radio-colloid particularly in low resource environments such as ours [9]. The chance of successful application of this technique in low resource environments such as ours is further enhanced because the learning curve for reliable use of this technique is very steep with less than a dozen cases required to achieve acceptable performance and because of the lower cost of methylene blue use without need for radiocolloid [10].

To date, there are no established non-surgical techniques for assessment of the axillary lymph nodes in invasive breast cancer. Physical exam has up to a $45 \%$ false negative rate, and imaging modalities such as magnetic resonance imaging, ultrasonography and positron emission tomography/computed tomography have limited use in terms of guiding therapy. Surgical staging is therefore the mainstay for assessment of the axilla.

SLNB is accomplished either by blue dye (isosulfan blue or methylene blue) or radioactive colloid injection (and subsequent gamma probe use). Isosulfan blue dye is associated with severe anaphylactic reactions which may require resuscitation in up to $1 \%$ of cases [11]. Methylene blue is regarded as a suitable alternative to isosulfan but this carries the risk of skin necrosis and local changes such as skin staining and erythema.

According to the landmark ACOSOG Z0011 (Alliance) trial, women with $\mathrm{T} 1$ or $\mathrm{T} 2$ invasive breast cancer, no palpable axillary adenopathy and only 1 or 2 sentinel lymph nodes with metastasis, the 10-year survival for those patients undergoing SLNB alone was comparable to those undergoing ALND [12]. Furthermore, in patients with one positive sentinel node and 2 or more adjacent negative axillary nodes the data suggest that further axillary dissection for additional lymph node sampling is not warranted [13].

These studies suggest that sentinel node biopsy continues to play an increasingly important role in management of the axilla in patients with early breast cancer, a major effect being a decreased use of more extensive potentially more harmful surgery while not compromising the standard of care.

\section{Conclusion}

Our local experience suggests SLNB using methylene blue dye is a reliable alternative to costlier and less available radioactive colloid/ gamma probe. In low resource settings such as ours, the methylene blue dye technique can be mastered with a very short learning period and should be considered for wider utilization in such environments. 


\section{References}

1. Walker RA, Hanby A, Pinder SE, Thomas J, Ellis IO (2012) Current issues in diagnostic breast pathology. J Clin Pathol 65: 771-785. [Crossref]

2. Gurleyik G, Gurleyik E, Aktekin A, Aker F (2015) Preoperative assessment of the axilla by surgeon performed ultrasound and cytology in patients with breast cancer. $J$ Clin Med Res 7: 440-445.

3. Canavese G, Catturich A, Vecchio C, Tomei D, Gipponi M, et al. (2009) Sentinel node biopsy compared with complete axillary dissection for staging early breast cancer with clinically negative lymph nodes: Results of randomized trial. Ann Oncol 20: 10011007. [Crossref]

4. Brogi E, Torres-Matundan E, Tan LK, Cody HS $3^{\text {rd }}$ (2005) The results of frozen section, touch preparation, and cytological smear are comparable for intraoperative examination of sentinel lymph nodes: A study in 133 breast cancer patients. Ann Surg Oncol 12: 173-180. [Crossref]

5. Mansel RE, Fallowfield L, Kissin M, Goyal A, Newcombe RG, et al. (2006) Randomized multicenter trial of sentinel node biopsy versus standard axillary treatment in operable breast cancer: The almanac trial. J Natl Cancer Inst 98: 599 609. [Crossref]

6. Morton DL, Wen D, Wong JH, Economou JS, Cagle LA, et al. (1992) Technical Details of Intraoperative Lymphatic Mapping for Early Stage Melanoma. Arch Surg 127: 392399. [Crossref]
7. Giuliano AE, Kirgan DM, Guenther JM, Morton DL (1994) Lymphatic mapping and sentinel lymphadenectomy for breast cancer. Ann Surg 220: 391-398. [Crossref]

8. Brouwer OR, van der Poel HG, Bevers RF, van Gennep EJ, Horenblas S (2016) Beyond penile cancer, is there a role for sentinel node biopsy in urological malignancies? Clin Transl Imaging 4: 395-410. [Crossref]

9. Fattahi AS, Tavassoli A, Rohbakhshfar O, Sadeghi R, Abdollahi A, et al. (2014) Can methylene blue dye be used as an alternative to patent blue dye to find the sentine lymph node in breast cancer surgery? J Res Med Sci 19: 918-922. [Crossref]

10. East JM, Valentine CS, Kanchev E, Blake GO (2009) Sentinel lymph node biopsy for breast cancer using methylene blue dye manifests a short learning curve among experienced surgeons: a prospective tabular cumulative sum (CUSUM) analysis. BMC Surg 9: 2. [Crossref]

11. Ozdemir A, Mayir B, Demirbakan K, Oygür N (2014) Efficacy of Methylene Blue in Sentinel Lymph Node Biopsy for Early Breast Cancer. J Breast Health 10: 88-91. [Crossref]

12. Giuliano AE, Ballman KV, McCall L, Beitsch PD, Brennan MB, et al. (2017) Effect of axillary dissection vs no axillary dissection on 10 -year overall survival among women with invasive breast cancer and sentinel node metastasis: the acosog z0011 (alliance) randomized clinical trial. JAMA 318: 918-926. [Crossref]

13. Ban EJ, Lee JS, Koo JS, Park S, Kim SI, et al. (2011) How many sentinel lymph nodes are enough for accurate axillary staging in t1-2 breast cancer? J Breast Cancer 14 296-300. [Crossref]

Copyright: (C2019 Sookar N. This is an open-access article distributed under the terms of the Creative Commons Attribution License, which permits unrestricted use, distribution, and reproduction in any medium, provided the original author and source are credited. 